\title{
Mishaps in the Washroom-A Rare Case
}

Neelu Desai ${ }^{1^{*}}$ and Avinash Desai ${ }^{2}$

${ }^{1}$ Division of Pediatric Neurology, PD Hinduja National Hospital \& Medical Research Centre, Veer Savarkar Marg, Mahim, Mumbai-400016, India

${ }^{2}$ Department of Pediatrics, Shushrusha Hospital, Dadar, Mumbai-400028, India

*Corresponding author: Neelu Desai, Division of Pediatric Neurology, PD Hinduja National Hospital \& Medical Research Centre, Veer Savarkar Marg, Mahim, Mumbai-400016, India, Tel: +91-22-39818181; E-mail: neeludesai@hotmail.com

Received date: September 10, 2016; Accepted date: October 13, 2016; Published date: October 20, 2016

Copyright: (c) 2016 Desai N, et al. This is an open-access article distributed under the terms of the Creative Commons Attribution License, which permits unrestricted use, distribution, and reproduction in any medium, provided the original author and source are credited.

\section{Introduction}

Seizures evoked by a specific stimulus are known as reflex seizures and comprise $5 \%$ of all epilepsies. The stimulus can be simple (flash of light) or more complex like reading, writing, doing arithmetic, or even thinking about specific topics [1]. Seizures occurring during micturition are rare and were first reported in 1964 by Zivin et al. [2] and subsequently by few other authors [3-12]. Here we report an interesting case of intractable seizures in a young girl consistently triggered by micturition.

\section{Case}

AK, a 6 year old girl presented with paroxysmal events during micturition for past 15 days. Each time on voiding, she would get bilateral lower limb jerking for 10-15 s followed by transient paralysis of both lower limbs with unresponsiveness. She had 10-12 such episodes daily and on two occasions, these events were followed by generalised tonic clonic jerking with loss of consciousness for five minutes followed by drowsiness. She was commenced on valproic acid (VA) by the treating paediatrician at $20 \mathrm{mg}$ per kg body weight with no benefit and subsequently was referred to our tertiary facility.

On probing details, all events occurred only during voiding but not otherwise. Child had a normal birth history but development was delayed for which she was never evaluated. She walked after 2 years and spoke first words only at 2.5 years. Her speech was not very comprehensible. She was not able to read or write alphabets in grade 1. Her head size was normal with normal physical examination. Her formal IQ test could not be done due to non-cooperation but her Social Quotient on Vineland Social Maturity Scale was 56 suggestive of mild sub-normality. Both parents did not report any significant mental or physical illnesses and mother's behaviour during or prior to pregnancy was normal. She had two elder siblings, both of whom were normal.

Differentials considered for micturition related events were micturition reflex epilepsy or micturition syncope or ictal urinary urge of non- dominant temporal lobe epilepsy. However, high frequency of events occurring each time on micturition and the semiology of events made the latter two unlikely.

Interictal EEG showed left parietal and midline frontoparietal epileptic discharges with mild focal fast activity in left parietal region (P3) in sleep state.(Figures 1a and 1b) Video EEG during micturition was performed. Within $10 \mathrm{~s}$ of micturition, the child developed bilateral lower limb and pelvic jerking with vocalisation followed by unresponsiveness. The entire event lasted $15 \mathrm{~s}$ and the child was responsive immediately after the event. The ictal EEG showed a rhythmic discharge starting from the midline parietal region $(\mathrm{Pz})$ spreading rapidly to bilateral parasagittal leads (Figures $2 \mathrm{a}$ and $2 \mathrm{~b}$ ). Diagnosis of micturition induced reflex epilepsy was confirmed and the common entity of micturition syncope was excluded. A 3 tesla MRI brain with thin cuts from midline frontoparietal region was performed which was normal. An interictal PET scan was done to rule out an occult dysplasia. It showed mild hypometabolism in left parietal lobe posteriorly and left inferior temporal lobe but was not confirmatory and needed ictal study to correlate.

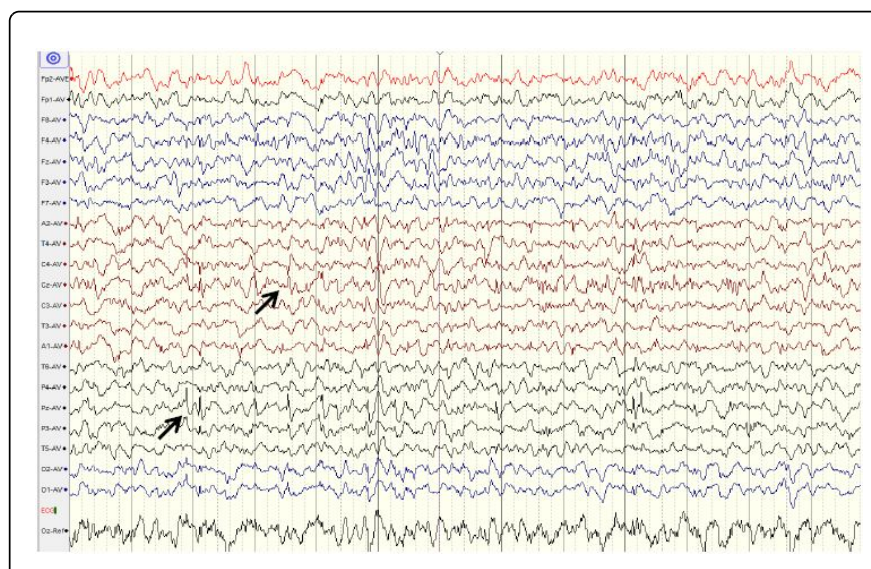

Figure 1: Interictal EEG shows midline central and parietal spikes.

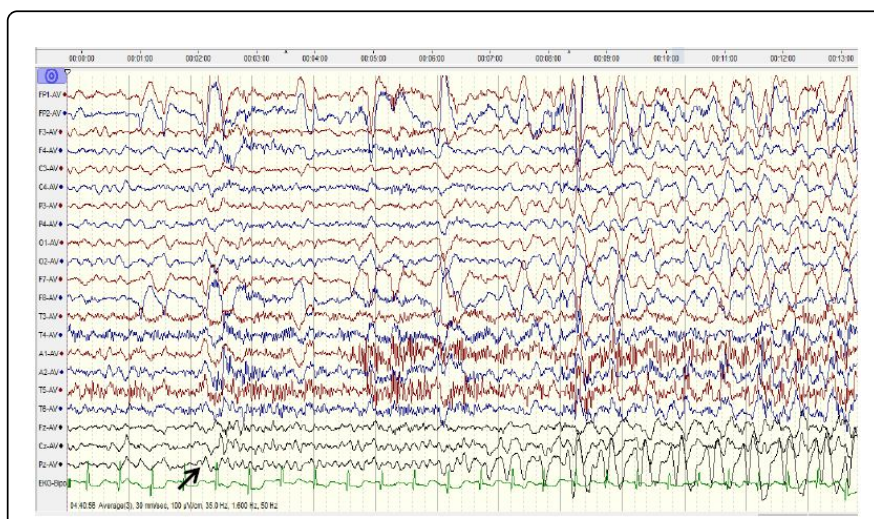

Figure 2: Ictal EEG on common average montage shows a distinct fast rhythm starting from $\mathrm{Pz}$ and $\mathrm{Cz}$ leads.

Oxcarbamazepine (OXC) was started followed by clobazam (CLB) which led to seizure freedom for a month. On tapering sodium valproate, seizures recurred and persisted despite restarting it. Leviteracetam (LVT) worsened the events hence Lamotrigine (LTG) 
was commenced. Seizures were controlled on four antiepileptic drugs (VA-30 mk/kg/d, OXC-40 mg/kg/d, CLB-1 mg/kg/d, LTG-2 mg/kg/d) for 2 months with a plan to slowly taper two of them subsequently.

After 2 months, seizures recurred and this time were also provoked by defecation and bathing besides micturition. She also had two spontaneous seizures where no trigger could be identified. Dose of lamotrigine was increased upto $5 \mathrm{mg} / \mathrm{kg} / \mathrm{d}$. Though seizures decreased to one per month from daily events, she is still not seizure free for last 6 months. A plan of ketogenic diet has been discussed with the family. This is a high fat, adequate-protein, low-carbohydrate diet that forces body to burn fats than carbohydrates and generates ketones which have anti-epileptic activity.

\section{Discussion}

Reflex epilepsies are a group of rare epilepsy syndromes where a particular stimulus-external or internal induces seizures. The usual etiologies are genetic or acquired in nature [1]. Focal brain lesions or occult focal cortical dysplasias can evoke reflex seizures. Pure reflex epilepsies have seizures exclusively in response to stimuli and do not suffer spontaneous seizures. Possible mechanism involves regions of cortical hyperexcitability that overlap with areas physiologically activated during specific cognitive or motor or sensory tasks [13].

Micturition reflex epilepsy is an extremely rare form of reflex epilepsy with not more than 12 cases described in literature according to our knowledge [2-12]. Passing urine is the triggering stimulus in all though the exact trigger could be somatosensory, viscerosensory, olfactory or auditory [14].

All cases have had onset in childhood (6 to 14 years) with no sex predilection [2-12]. Developmental delay as in our case was seen in some of them which could suggest pre-existing brain impairment. High prevalence of coexisting spontaneous seizures was also seen in patients with reflex seizures. Higuchi et al. [9] reported a child with seizure induced by both micturition and defecation.

EEGs in all patients with micturition reflex epilepsy have consistently shown a midline central $(\mathrm{Cz})$ or frontal focus as in our case which corroborates with a symptomatic zone in the supplementary motor area (SMA) [3-12]. This suggests that micturition induced seizures arise from the SMA, which controls both defecation and urination. MRI brains of all reported patients with micturition reflex epilepsy were either normal or showed nonspecific changes which could not be attributed as the seizure focus. A single case report mentioned presence of a calcified granuloma in right frontal lobe as the possible seizure focus [4]. A possible focal area of cortical dysplasia could account for micturition reflex epilepsy however no definite confirmation has been made in any case.

Micturition is a complex task coordinated by multiple levels of the central and peripheral nervous system $[15,16]$. With help of Positron emission tomography (PET) studies, Blok et al. [17] concluded that the SMA is one of the regions physiologically activated during micturition/ defecation by contraction of the pelvic floor muscles. Using SPECT and functional MRI, Fukuyama et al. [15] and Bittorf et al. [18] also showed respectively that the SMA was involved in sphincter function during micturition/defecation. PET studies have shown that the rightsided micturition centres are more active than those on the left [17]. On Ictal SPECT study Glass et al. [7] reported hyperperfusion over the anterior cingulate gyrus and anterolateral right frontal lobe while Okumura et al. [8] found increased blood flow in the mesial frontal regions. The role of these regions in the cortical control of micturition is well known.

Though no standard antiepileptic drug (AED) regime is found highly efficacious in reflex seizures, most authors report a good response to conventional antiepileptic drugs. This was also noted in most cases with micturition reflex epilepsies who achieved remission with different drugs. The child reported by Whitney et al. [12] responded well to Lacosamide and clobazam, while Seth JH10 reported a good response to Lamotrigine monotherapy $(75 \mathrm{mg} /$ day). Higuchi T9 reported a good response to combination of clobazam (11 $\mathrm{mg} /$ day) and phenytoin $(120 \mathrm{mg} /$ day); the latter was also effective in the case reported by Okumara [8]. Our patient persisted to have infrequent seizures despite adequate doses of a combination of four AEDS similar to the patient reported by Glass HC7 who had a poor response to adequate trials of multiple AEDs (phenobarbital, valproic acid, clonazepam, topiramate, lamotrigine, clobazam) as well as ketogenic diet. Ketogenic diet was planned in our case as parents refused to try more drugs. .

\section{Conclusion}

Micturition-induced reflex epilepsy is rare form of reflex epilepsy with onset in childhood and is a differential diagnosis for micturition syncope. Video-EEG monitoring with provoking trigger could establish the diagnosis early in our case. A possible cortical dysplasia in supplementary motor area could be the underlying etiology though high resolution MRI was normal. Most cases of micturition-induced reflex epilepsy respond to standard antiepileptic drugs.

\section{References}

1. Ritaccio AL (1994) Reflex seizures. Neurol Clin 12: 57-83.

2. Zivin I, Rowley W (1964) Psychomotor epilepsy with micturition. Arch Intern Med 113: 8-13.

3. Yamatani M, Murakami M, Konda M, Konishi T, Suzuki Y, et al. (1987) An 8 year old girl with micturition-induced epilepsy. No To Hattatsu 19: 58-62.

4. Pradhan S, Kalita J (1993) Micturition induced reflex epilepsy. Neurol India 41: 221-223.

5. Ikeno T, Morikawa A, Kimura I (1998) A case of epileptic seizure evoked by micturition. Rinsho Nouha 40: 205-208.

6. Bourgeois BF (1999) A retarded boy with seizures precipitated by stepping into the bath water. Semin Pediatr Neurol 6: 151-157.

7. Glass HC, Prieur B, Molnar C, Hamiwka L, Wirrell E (2006) Micturition and emotion-induced reflex epilepsy: Case report and review of the literature. Epilepsia 47: 2180-2182.

8. Okumura A, Kondo Y, Tsuji T, Ikuta T, Negoro T, et al. (2007) Micturition induced seizures: Ictal EEG and subtraction ictal SPECT findings. Epilepsy Res 73: 119-121.

9. Higuchi T, Fukuyama T, Misawa Y, Inaba Y, Ichikawa M, et al. (2011) Reflex seizures induced by micturition and defecation, successfully treated with clobazam and phenytoin. Epileptic Disord 13: 166-171.

10. Seth JH, McLaughlin C, Eriksson S, Fowler CJ, Walker MC, et al. (2014) Blackouts in the toilet: A case of micturition-induced reflex epilepsy. Pract Neurol 14: 261-263.

11. Harbord MG, Mitchell C (2002) Reflex seizures induced by defecation, with an ictal EEG focus in the left frontotemporal region. Epilepsia 43: 946-947.

12. Whitney R, Callen DJ (2013) Micturition-induced seizures: A rare form of reflex epilepsy. Pediatr Neurol 49: 61-63.

13. Ferlazzo E, Zifkin BG, Andermann E, Andermann F (2005) Cortical triggers in generalized reflex seizures and epilepsies. Brain 128: 700-710. 
14. Bass N, Wyllie E, Comair Y, Kotagal P, Ruggieri P, et al. (1995) Supplementary sensorimotor area seizures in children and adolescents. J Pediatr 126: 537-544.

15. Fukuyama H, Matsuzaki S, Ouchi Y (1996) Neural control of micturition in man examined with single photon emission computed tomography using 99mTc-HMPAO. Neuroreport 7: 3009-3012.

16. Fowler CJ, Griffiths D, de Groat WC (2008) The neural control of micturition. Nat Rev Neurosci 9: 453-466.
17. Blok BF, Sturms LM, Holstege G (1997) A PET study on cortical and subcortical control of pelvic floor musculature in women. J Comp Neurol 389: 535-544.

18. Bittorf B, Ringler R, Forster C, Hohenberger W, Matzel KE (2006) Cerebral representation of the anorectum using functional magnetic resonance imaging. Br J Surg 93: 1251-1257. 